\title{
Condom-Assisted Transurethral Resection: A New Surgical Technique for Urethral Tumor
}

\author{
Kazuhiro Sato, Motofumi Suzuki", Masayoshi Nagata, Hiroaki Nishimatsu, \\ Akira Ishikawa, Yasuhiko Igawa, Haruki Kume, Yukio Homma \\ Department of Urology, Graduate School of Medicine, The University of Tokyo, Tokyo, Japan \\ E-mail: suzukim-uro@h.u-tokyo.ac.jp \\ Received August 9, 2010; revised August 9, 2010; accepted August 9, 2010
}

\begin{abstract}
Backgrounds: It is difficult to develop clear endoscopic field during transurethral resection (TUR) of urethral tumors, because irrigation water easily spills out from the meatus. We have devised a surgical technique to facilitate TUR of urothelial tumors. Methods: A 79-year old man developed urethral tumors in the anterior urethra and fossa navicularis 6 months after TUR of bladder tumor. We covered his penis and the resectoscope with a tip-cut condom. The tip-end of condom and the resectoscope sheath was tightly sealed and irrigation water was filled inside the condom. Results: The urethral tumors were successfully resected under a clear vision by filling irrigation water inside a tip-cut condom that covered over the resectoscope and the penis. Conclusion: This technique may be useful for TUR of anterior urethral tumors, especially tumors at fossa navicularis, which is often associated with difficulty in obtaining clear surgical vision.
\end{abstract}

Keywords: Urethral Tumor, Fossa Navicularis, TUR, Condom

\section{Introduction}

Urethral cancer develops primarily or secondary to bladder carcinoma. Overall risk of urethral recurrence of urothelial carcinoma in men after radical cystectomy for bladder cancer is approximately 4 to $10 \%$ [1-3]. Urethral recurrence is less frequent after transurethral resection of bladder tumor (TUR-Bt); Saika et al. found a urethral recurrence rate of $2.6 \%$ in 303 patients with superficial bladder cancer undergoing TUR-Bt [4].

However, endoscopic surgery for urethral tumors, especially tumors at fossa navicularis may not be easy. We experienced recurrent urethral tumors involving fossa navicularis after TUR-Bt and managed them by a new TUR procedure.

\section{Methods}

\subsection{Case Report}

A 79-year old man was admitted to our hospital for the left acute pyelonephritis. During the check-up a single papillary tumor was detected in the bladder, which was subsequently treated by TUR-Bt on 25th September 2008. Pathological finding was grade 1 urothelial carcinoma,
pTa. He was followed by cystoscopy every three months and found for urethral tumors in the anterior urethra and fossa navicularis on 27th April 2009 (Figure 1).

\section{Findings}

\subsection{A New TUR Procedure - Condom - Assisted TUR of Urethral Tumor}

On 21 st July 2009, endoscopic resection of the urethral tumors was attempted. First we performed conventional TUR by holding the resectoscope tip at the urethral meatus. However it was almost impossible to endoscopically visualize the tumors, especially tumors at fossa navicularis, because irrigation water easily spilled out from the meatus. Then we covered his penis and the resectoscope with a tip-cut condom.

The tip-end of condom and the resectoscope sheath was tightly sealed and the proximal end was compressed manually to prevent water leakage. Irrigation water was filled inside the condom at 60 to $80 \mathrm{cmH}_{2} \mathrm{O}$ (Figure 2). By this procedure, we could perform TUR of urethral tumors under a clear view of the urethral lumen. We resected all the tumors to the depth of submucosa and coagulated their trace to stop bleeding. No recurrent tumors 


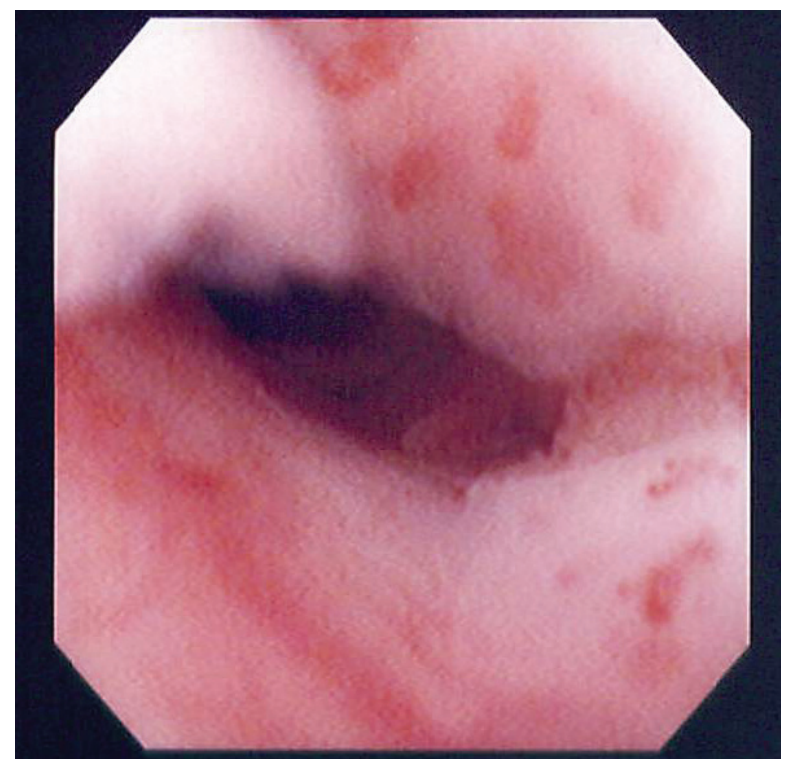

(a)

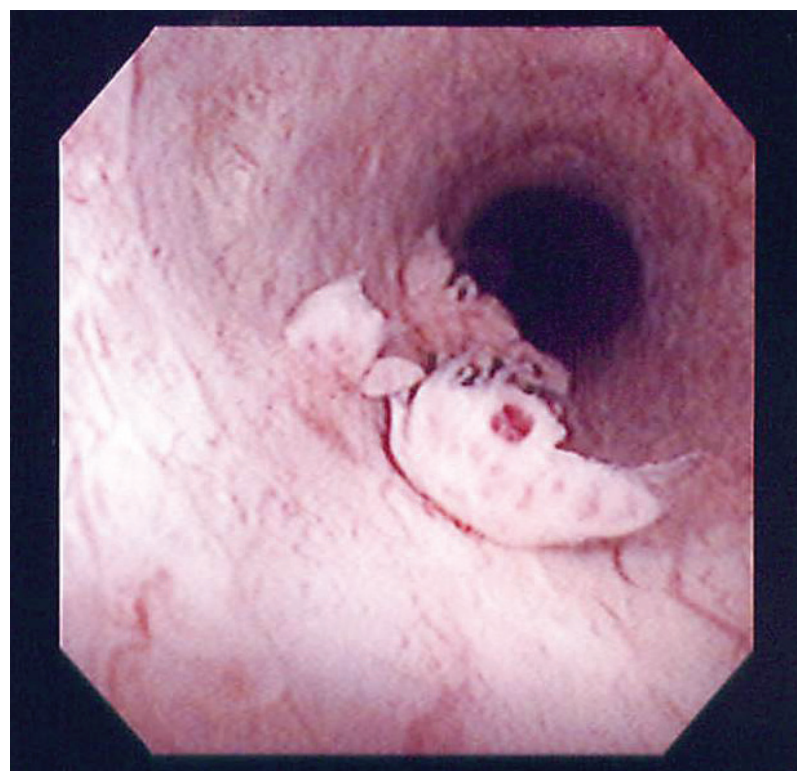

(b)

Figure 1. (a) Superficial tumors of fossa navicularis; (b) Superficial tumors of anterior urethra.

were found in the bladder. Pathological finding of urethral tumors was grade 2 urothelial carcinoma with squamous differentiation, $\mathrm{pTa}$. He was free of recurrence in the urinary tract one year postoperatively.

\section{Results and Discussion}

Urethral cancer, primary or recurrent, is an uncommon disease associated with poor outcomes. Steele, et al. reviewed 11 cases of primary urethral tumor of fossa navicularis [5]. Four men with superficial urethral tumors underwent TUR. A single case was free of disease one

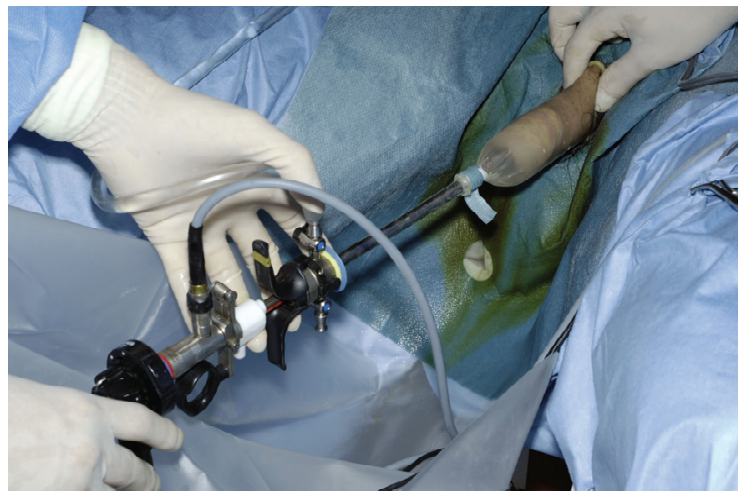

Figure 2. Resectoscope is inserted through a tip-cut condom. Proximal end of condom is manually fixed and the connection between condom and resectoscope was tightly sealed. Irrigation water filled in the condom enables to visualize tumors at fossa navicularis by urethroscopy.

year after TUR, while the remaining three were not reported for the prognosis. Seven men with invasive urethral tumor received total penectomy, partial penectomy or radiation. Saika et al. reported that four of five men with recurrent superficial urethral tumor treated by TUR died of tumor progression [4]. Yoshida et al. performed salvage urethrectomy in three of four men who developed recurrent urethral tumor after TUR of superficial urethral tumors [6]. Poor prognosis of urethral tumors may be related to the fact that the lamina propria is the only barrier between the urethral mucosa and the vascular corpora [7]. Endoscopic surgery for urethral tumors is a challenge, especially tumors at fossa navicularis due to the poor surgical field. Excision and fulguration under direct vision is commonly used for eradicating urethral tumors at the fossa navicularis $[8,9]$. Our new TUR procedure using tip-cut condom may be useful to visualize urethral tumors in the anterior urethra or fossa navicularis and manage them properly.

\section{Conclusions}

We have devised a surgical technique to facilitate TUR of urothelial tumors in fossa navicularis by using a tipcut condom that covers the resectoscope and the penis.

\section{Acknowledgements}

No conflict of interest.

\section{References}

[1] J. A. Freeman, D. Esrig, J. P. Stein and D. G. Skinner, "Management of the Patient with Bladder Cancer. Urethral Recurrence," Urologic Clinics of North America, Vol. 21, No. 4, 1994, pp. 645-651. 
[2] J. P. Stein, P. Clark, G. Miranda, et al., "Urethral Tumor Recurrence Following Cystectomy and Urinary Diversion: Clinical and Pathological Characteristics in 768 Male Patients," Journal of Urology, Vol. 173, No. 4, 2005, pp. 1163-1168.

[3] K. S. Cho, J. W. Seo, Y. H. Lee, et al., "The Risk Factor for Urethral Recurrence after Radical Cystectomy in Patients with Transitional Cell Carcinoma of the Bladder," Urology International, Vol. 82, No. 3, 2009, pp. 306-311.

[4] T. Saika, T. Tsushima, Y. Nasu, et al., “Anterior Urethral Recurrence of Superficial Bladder Cancer: Its Clinical Significance," Acta Medica Okayama, Vol. 57, No. 6, 2003, pp. 293-297.

[5] G. S. Steele, J. R. Fielding, A. Renshaw, et al., "Transitional Cell Carcinoma of the fossa Navicularis," Urology, Vol. 50, No. 5, 1997, pp. 792-795.
[6] K. Yoshida, H. Nishiyama, H. Kinoshita, et al., "Surgical Treatment for Urethral Recurrence after Ileal Neobladder Reconstruction in Patients with Bladder Cancer," BJU International, Vol. 98, No. 5, 2006, pp. 1008-1011.

[7] D. P. Hickey, M. S. Soloway and W. M. Murphy, "Selective Urethrectomy Following Cystoprostatectomy for Bladder Cancer," Journal of Urology, Vol. 136, No. 4, 1986, pp. 828-830.

[8] R. A. Mevorach, L. R. Cos, P. A. di Sant'Agnese and M. Stoler, "Human Papilloma Virus Type 6 in Grade 1 Transitional Cell Carcinoma of the Urethra," Journal of Urology, Vol. 143, No. 1, 1990, pp. 126-128.

[9] L. H. Stewart and S. R. Johnston, "Transitional Cell Carcinoma of the Fossa Navicularis," British Journal of Urology, Vol. 72, No. 1, 1993, pp. 121-122. 